\title{
Deformation Measurement of Membrane Structure Based on Fiber Sensor Array
}

\author{
Jie Zou ${ }^{*}$, Dongxu Li \\ National University of Defense Technology, Changsha, Hunan, China \\ *zoujie_nudt@163.com
}

Keywords: membrane structure; fiber sensor array; deformation measurement

\begin{abstract}
In order to solve the problem of deformation measurement of membrane structure, the method based on fiber senor array is proposed. Firstly, FBG (Fiber Bragg Grating) sensors are used to get the strain distribution of the structure's surface, and furthermore to obtain the curvature distribution. Secondly, the surface is reconstructed through a kind of surface reconstruction algorithm based on curvature, and then we optimize the configuration of FBG sensor to improve the accuracy. Finally, the practicability of the method is proved through simulation. The results of simulation also indicate its high measuring accuracy.
\end{abstract}

\section{Introduction}

Membrane structure is widely used in the field of aerospace, such as solar panels in satellites and membrane SAR antenna. Membrane structure will inevitably deform due to complex space environment, and thus its capability will be influenced. On the one hand, membrane structure has the features of low stiffness and light weight; on the other hand, the space environment where membrane structure works in is very special. The structure can be influenced by high and low temperature, sunray pressure and gravity gradient which come from external disturbance, and satellite attitude adjustment, orbit maneuver and other disturbance inside of satellites or aircraft. Once the membrane structure is deformed, its performance will deteriorate. Therefore, we have to control its deformation.

The premise of deformation control for the structure is precise deformation measurement, and it's not an easy job. There are two main methods which are contact measurement and non-contact one. Optical photogrammetry as the non-contact method possesses the advantages of high accuracy and not influencing the objective, but it can hardly be adopted in space systems because it needs to be high enough above the objective to measure it. The representatives of contact measurement are traditional electric sensors and fiber sensors. Electric sensors are too heavy for membrane structure, and in contrast, fiber sensors are light enough to not influence the structure.

Using fiber sensors for measuring has many merits and many researchers are interested. Haiwei Xu applied distributed FBG sensors to strain monitoring of morphing wings' structure [1]. Jun Yang researched distributed fiber-optic white light interferemetric deformation measuring technique [2]. Stephan Rapp used FBG sensors in dynamic shape estimation by modal approach [3]. Xiaojin Zhu did the research on shape reconstruction of FBG intelligent flexible structure based on B-spline fitting [4]. Martin Schroeck adopted FBG sensor arrays in strain monitoring in steel rock bolts [5]. However, there are few researches and reports of applying fiber sensors to deformation measurement of membrane structure. This paper presents a method based on fiber sensor array, and it will be proved by simulation.

\section{The method of deformation measurement}

FBG sensors can accurately measure strain. Albert Bosse used FBG sensors to map strain-displacement of a precision truss and the results turned out good [6].We can get the strain distribution of structures by fiber sensor array composed of FBG sensors. Then, the curvature 
distribution can be deducted. In the end, we can reconstruct the surface and deformation measurement is fulfilled. There are many surface reconstruction algorithms for now. Fengsong Zheng raised a way of B spline curve and surface reconstruction based on genetic algorithm [7]. Elena Franchini studied the implicit shape reconstruction of unorganized points using PDE-Based deformable 3D manifolds [8]. Gaofeng Meng did a research on curve and surface Reconstruction from unorganized points [9]. Lincong Fang presented a method of radial basis functions based surface reconstruction algorithm [10]. Ruud M Bolle researched three-dimensional surface reconstruction methods [11].

2.1 The method of measuring structures' curvature based on FBG sensors. Supposing that FBG adheres to Hooke's law, and there is no shear strain, and furthermore FBG's refractive index distributes uniformly on the cross section, when the temperature stays unchanged, FBG's change of central reflection wavelength $\Delta \lambda_{B}$ and strain $\varepsilon$ at the measuring point meets:

$$
\frac{\Delta \lambda_{B}}{\lambda_{B}}=\left(1-P_{e}\right) \varepsilon
$$

Where $\lambda_{B}$ is the central reflection wavelength of FBG, $P_{e}$ is the fiber's valid elastic-optic constant.

Under the condition of pure bending, for the flexible uniform section truss, we choose an infinitesimal from the structure, whose length is $L$ and thickness is $h$ as shown in Fig. 1. In Fig. 1, the dotted line is the neutral layer. $\Delta L$ is the change of the length of infinitesimal. $\theta$ is the central angle when the infinitesimal deforms. $M$ is the moment.
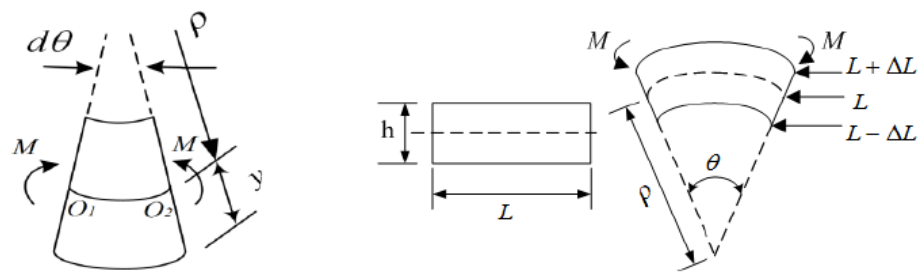

Figure 1. Infinitesimal's deformation of pure bending

From Fig. 1 we can get

$L=\rho \theta$

And

$L+\Delta L=(\rho+h / 2) \theta$

Substitution of Eq. (2) into Eq. (3) yields

$k=\frac{1}{\rho}=\frac{2}{h} \frac{\Delta L}{L}=\frac{2}{h} \varepsilon$

Where $k$ is the curvature, $\varepsilon$ is the strain expressed as

$\varepsilon=\frac{\Delta L}{L}$

Substitution of Eq. (1) into Eq.(4) yields

$k=\frac{2 \Delta \lambda_{B}}{\lambda_{B}\left(1-P_{e}\right) h}=F \Delta \lambda_{B}$ 
From Eq. (6) we can know that curvature $k$ and FBG's change of central reflection wavelength $\Delta \lambda_{B}$ are in direct ratio.

2.2 Surface reconstruction algorithm based on curvature. Curved surface can be seen as a set of countless curves. The reconstruction of surface can be accomplished by reconstructing several curves and fitting them smoothly. Firstly, we can get every point's coordinates through curvature, and furthermore reconstruct curves. Then by fitting curves smoothly, the surface is reconstructed.

Jincong Yi raised linear corner cut recursive algorithm based on curvature [12], and in order to increase the accuracy, this paper improved this algorithm and get quadratic-function corner cut recursive algorithm. For curves, once two points in the curve are close enough, the arc between them can be regarded as arc infinitesimal. As shown in Fig. 2, assuming that the starting point of arc infinitesimal $\Delta s_{n}$ is $O_{n}$ and the terminal point is $O_{n+1}$, where the curvature is $k_{n}$ and $k_{n+1}$, and corresponding coordinates are $\left(x_{n}, y_{n}\right)$ and $\left(x_{n+1}, y_{n+1}\right) ; \alpha_{n}$ and $\alpha_{n+1}$ are tangent vectors at the point of $O_{n}$ and $O_{n+1} ; l_{n}$ is the corresponding chord length of $\Delta s_{n} ; \theta_{n}$ and $\theta_{n+1}$ are the included angel of $\alpha_{n}, \alpha_{n+1}$ and axis $x ; \Delta \theta_{n}$ is the center angel.

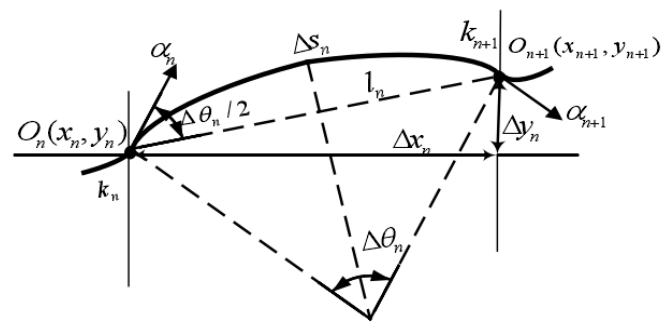

Figure 2. Analysis of arc infinitesimal

From Fig. 2 we can get

$$
\left\{\begin{array}{l}
\Delta \theta_{n}=\theta_{n+1}-\theta_{n} \\
l_{n}=2 \sin \left(\Delta \theta_{n} / 2\right) / k_{n},\left(k_{n} \neq 0\right) \\
l_{n}=\Delta s_{n} \\
\Delta x_{n}=l_{n} \cdot \cos \left(\theta_{n}-\Delta \theta_{n} / 2\right) \\
\Delta y_{n}=l_{n} \cdot \sin \left(\theta_{n}-\Delta \theta_{n} / 2\right) \\
x_{n+1}=x_{n}+\Delta x_{n} \\
y_{n+1}=y_{n}+\Delta y_{n}
\end{array}\right.
$$

The definition of curvature yields

$$
\theta(s)=\int k(s) d s
$$

Jincong Yi supposed that curvature $k$ and arc $s$ are in direct ratio in his paper, but in order to use more curvatures of different points to increase accuracy, this paper assumes that $k$ is the quadratic function of $s$, which can be expressed as

$$
k=a \times s^{2}+b \times s+c
$$

Where $a, b$ and $c$ are constants. The curve is composed of many arcs from $O_{1} \sim O_{2}$ to $O_{n-1} \sim O_{n}$. For two arcs between $O_{n}$ and $O_{n+2}$ 


$$
\left\{\begin{array}{l}
k_{n}=a \times s_{n}^{2}+b \times s_{n}+c \\
k_{n+1}=a \times s_{n+1}^{2}+b \times s_{n+1}+c \\
k_{n+2}=a \times s_{n+2}^{2}+b \times s_{n+2}+c
\end{array}\right.
$$

Solution of Eq. (10) is

$$
\left\{\begin{array}{c}
a=\frac{\left(k_{n+2}-k_{n}\right)\left(s_{n+1}-s_{n}\right)-\left(k_{n+1}-k_{n}\right)\left(s_{n+2}-s_{n}\right)}{\left(s_{n+1}-s_{n}\right)\left(s_{n+2}^{2}-s_{n}^{2}\right)-\left(s_{n+2}-s_{n}\right)\left(s_{n+1}^{2}-s_{n}^{2}\right)} \\
b=\frac{\left(k_{n+2}-k_{n}\right)\left(s_{n+1}^{2}-s_{n}^{2}\right)-\left(k_{n+1}-k_{n}\right)\left(s_{n+2}^{2}-s_{n}^{2}\right)}{\left(s_{n+2}-s_{n}\right)\left(s_{n+1}^{2}-s_{n}^{2}\right)-\left(s_{n+1}-s_{n}\right)\left(s_{n+2}^{2}-s_{n}^{2}\right)} \\
c=k_{n}-\frac{k_{n+2}\left(s_{n}^{2} s_{n+1}+s_{n} s_{n+1}^{2}-2 s_{n}^{3}\right)-k_{n+1}\left(s_{n}^{2} s_{n+2}+s_{n} s_{n+2}^{2}-2 s_{n}^{3}\right)+k_{n}\left(s_{n}^{2} s_{n+2}+s_{n} s_{n+2}^{2}\right)}{\left(s_{n+1}-s_{n}\right)\left(s_{n+2}^{2}-s_{n}^{2}\right)-\left(s_{n+2}-s_{n}\right)\left(s_{n+1}^{2}-s_{n}^{2}\right)}
\end{array}\right.
$$

Substitution of Eq. (9) into Eq. (8) yields

$$
\theta(s)=\frac{1}{3} \cdot a \times s^{3}+\frac{1}{2} b \times s^{2}+c \times s+d
$$

Eq. (12) is a function of $s$, and its constant $d$ can be got by using boundary condition. Because every curvature of every arc can be calculated by interpolating finite dispersed curvature measured by FBG sensors, there is a solution of Eq. (12), and moreover we can get coordinates of every point through Eq. (7). There are many ways of interpolation such as linear interpolation, cubic polynomial interpolation and spline interpolation.

\section{Optimization of FBG sensors' configuration}

Although FBG sensors can be multiplex and therefore we can have plenty sensors on structures without influencing structures a lot, the configuration of sensors still has a great impact on results. Thus, we have to optimize the configuration.

3.1 Particle swarm optimization. Particle swarm optimization (PSO) initializes as a group of random particles (random solution), and searches the optimum solution through iteration. In every iteration, one particle updates itself by following two extremums: one is the extremum particle found by itself, which is called individual extremum; the other one is the optimum solution found by the population so far, which is called global extremum.

Assuming that in a targeted searching space of $D$ dimension, a population consists of $N$ particles. The position of $i^{t h}$ particle is

$$
X_{i}=\left(x_{i 1}, x_{i 2}, \cdots, x_{i D}\right), i=1,2, \cdots, N
$$

The $i^{\text {th }}$ particle's velocity is

$$
V_{i}=\left(v_{i 1}, v_{i 2}, \cdots, v_{i D}\right), i=1,2, \cdots, N
$$

The $i^{\text {th }}$ particle's individual extremum is

$$
P_{b e s t}=\left(p_{i 1}, p_{i 2}, \cdots, p_{i D}\right), i=1,2, \cdots, N
$$


The global extremum is

$$
g_{\text {best }}=\left(p_{g 1}, p_{g 2}, \cdots, p_{g D}\right)
$$

Once two extremums are found, one particle can update its position and velocity by

$$
\begin{aligned}
& v_{i d}=\omega v_{i d}+c_{1} r_{1}\left(p_{i d}-x_{i d}\right)+c_{2} r_{2}\left(p_{g d}-x_{i d}\right) \\
& x_{i d}=x_{i d}+v_{i d}
\end{aligned}
$$

Where $\omega$ is the inertia weight, $c_{1}$ and $c_{2}$ are learning factors, $r_{1}$ and $r_{2}$ are random numbers from $[0,1]$.

3.1 Criterion for optimization of configuration. Supposing that the membrane structure is a rectangle, we can divide it into equal division grid, and every node is the possible position of a sensor as shown in Fig.3.

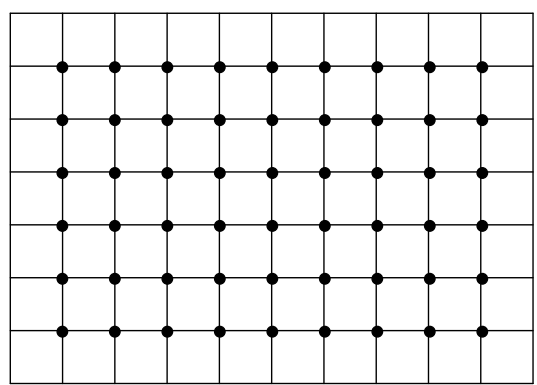

Figure 3. Possible positions of sensors

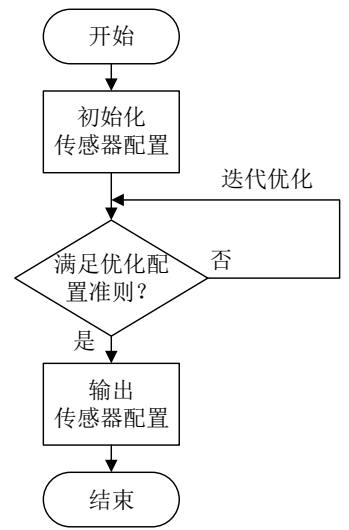

Figure 4. Flow chart of optimization

In order to increase the measurement accuracy for membrane structure, multiple FBG sensors should be used meanwhile. The criterion for optimization of configuration is expressed as

$$
\delta_{3 \sigma}=3 \sqrt{\frac{1}{I} \sum_{i=1}^{I}\left(\bar{Z}_{i}-Z_{i}\right)^{2}}
$$

Where $\delta_{3 \sigma}$ is called shape error, $I$ is the total number of sampling points on structure for calculating $\delta_{3 \sigma}, \bar{Z}_{i}$ is the displacement of axis $z$ of point $i$ coming from the results of surface reconstruction, while $Z_{i}$ coming from the results of simulation.

Obviously, the smaller $\delta_{3 \sigma}$ is, the more similar the reconstructed surface and real surface, and thus the accuracy is higher. The flow chart of optimization is shown as Fig. 4.

\section{Simulation and analysis}

The simulation model for this paper is shown as Fig. 5. The structure consists of two aluminum alloy sheets (red ones), two silica gel sheets (blue ones) and one membrane structure (yellow one). The external side of aluminum alloy and silica gel sheet is $600 \times 600 \mathrm{~mm}$ and the inner side is $400 \times 400 \mathrm{~mm}$, and two kinds of sheets' thickness is $2 \mathrm{~mm}$. The size of membrane is $600 \times 600 \mathrm{~mm}$ with thickness of $0.235 \mathrm{~mm}$. Four sheets are bounded by bolt. Two aluminum alloy sheets nip two silica gel sheets and two silica gel sheets nip the membrane. The properties of these materials are shown in Table 1. 

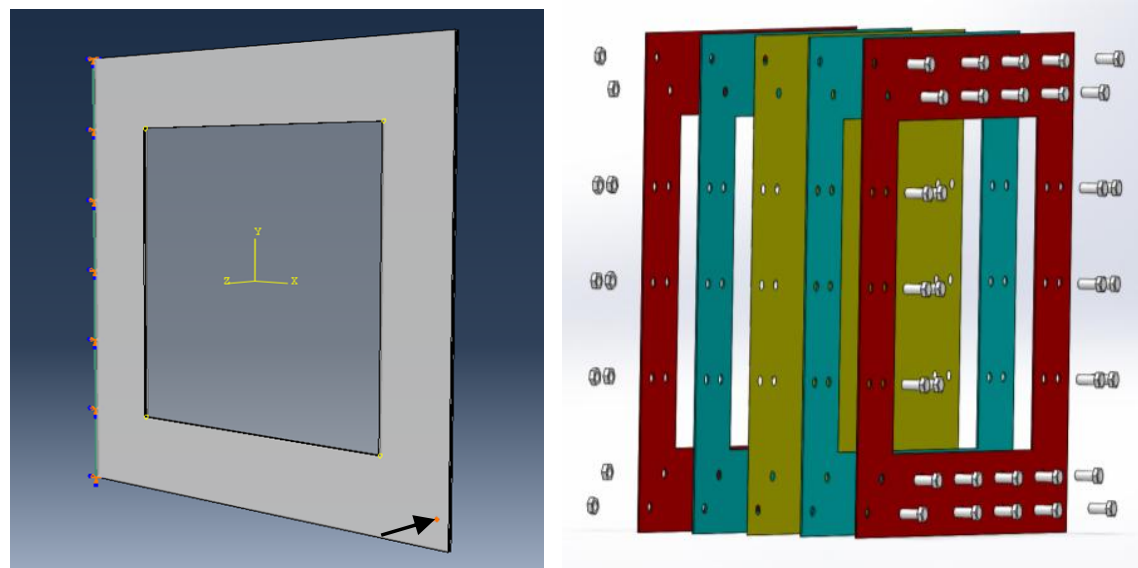

Figure 5 Simulation Model

Table 1. Properties of materials

\begin{tabular}{cccc}
\hline Material & Density $\left[\mathrm{kg} / \mathrm{m}^{3}\right]$ & $\begin{array}{c}\text { Elastic modulus } \\
{[\mathrm{Gpa}]}\end{array}$ & Poisson ration \\
\hline $\begin{array}{c}\text { Alumminium } \\
\text { alloy }\end{array}$ & $2.78 \times 10^{3}$ & 70 & 0.3 \\
\hline Silica gel & $1.2310^{3}$ & 1.2 & 0.48 \\
\hline Membrane & $1.1134 \times 10^{3}$ & 0.8 & 0.3 \\
\hline
\end{tabular}

In the optimization process, learning factors $c_{1}=1.5$ and $c_{2}=1.5$, while inertia weight $\omega=0.5$. When the number of sensors is $2 \times 2$, the optimum configuration of sensors is shown as Fig. 6 (Red dots represent the positions of sensors), and the comparison of reconstructed surface and deformation surface from ABAQUS simulation is shown as Fig. 7 (The red surface is from ABAQUS simulation and the blue one is reconstructed surface). The shape error $\delta_{3 \sigma}$ is $1.887 \mathrm{~mm}$.

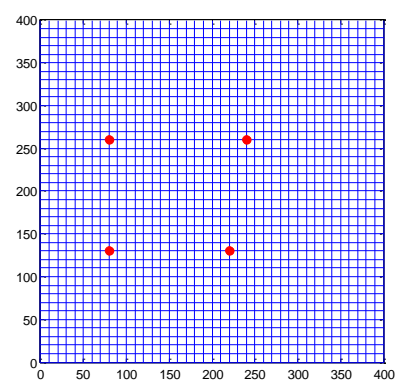

Figure 6. Optimum positions of $2 \times 2$ sensors

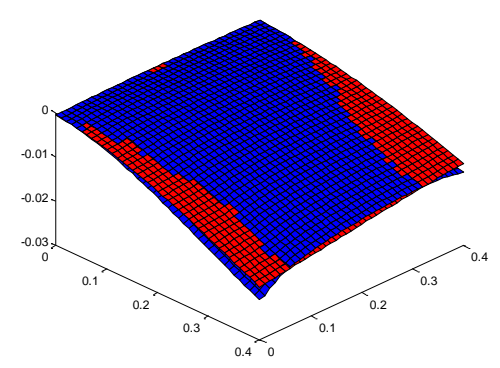

Figure 7. Result of comparison ( $2 \times 2$ sensors)

When the number of sensors is $3 \times 3$, the optimum configuration of sensors is shown as Fig. 8 (Red dots represent the positions of sensors), and the comparison of reconstructed surface and deformation surface from ABAQUS simulation is shown as Fig. 9(The red surface is from ABAQUS simulation and the blue one is reconstructed surface). The shape error $\delta_{3 \sigma}$ is $0.621 \mathrm{~mm}$.

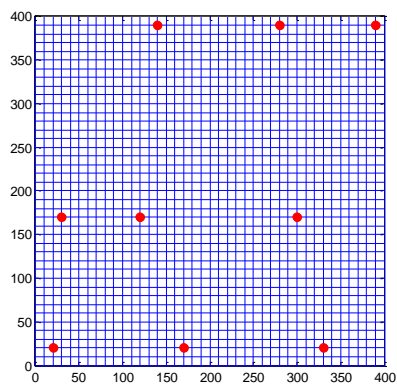

Figure 8 . Optimum positions of $3 \times 3$ sensors

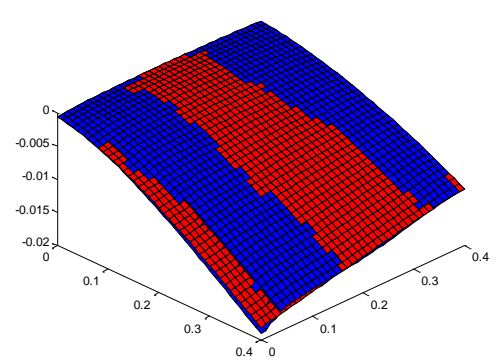

Figure 9. Result of comparison $(3 \times 3$ sensors $)$ 
When the number of sensors is $4 \times 4$, the optimum configuration of sensors is shown as Fig. 10 (Red dots represent the positions of sensors), and the comparison of reconstructed surface and deformation surface from ABAQUS simulation is shown as Fig. 11 (The red surface is from ABAQUS simulation and the blue one is reconstructed surface). The shape error $\delta_{3 \sigma}$ is $0.471 \mathrm{~mm}$.

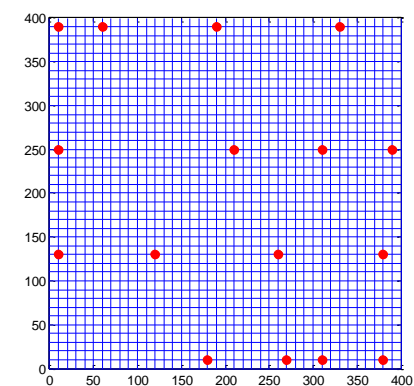

Figure 10. Optimum positions of $4 \times 4$ sensors

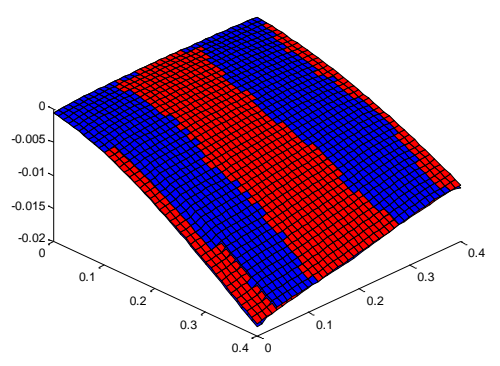

Figure 11. Result of comparison ( $4 \times 4$ sensors)

It can be seen from the results that the shape error $\delta_{3 \sigma}$ decreases sharply when number of sersors rises from 4 to 9 and modestly from 9 to 16 . Because that the more sensors, the more complex the system will be, we should use proper number of sensors as long as the accuracy is achieved.

Considering more number configurations of sensors, we can get different minimum shape errors as shown in Table 2.

Table 2. Minimum shape errors of different number of sensors

\begin{tabular}{cccccccc}
\hline Number of sensors & $2 \times 2$ & $3 \times 2$ & $2 \times 3$ & $3 \times 3$ & $4 \times 3$ & $3 \times 4$ & $4 \times 4$ \\
\hline $\begin{array}{c}\text { Minimun shape error } \\
{[\mathrm{mm}]}\end{array}$ & 1.887 & 0.713 & 1.856 & 0.621 & 0.518 & 0.617 & 0.471 \\
\hline
\end{tabular}

From Table II we can see that the number of vertical sensors have bigger influence on shape errors than lateral ones.

At the same time, different methods of interpolation also have different shape errors. For the optimum configuration of $4 \times 4$ sensors, the shape errors adopting different interpolation methods are shown in Table 3. It's obvious that spline interpolation is the best.

Table 3. Minimum shape errors of different interpolation methods

\begin{tabular}{cccc}
\hline Interpolation methods & Linear interpolation & $\begin{array}{c}\text { Cubic polynominal } \\
\text { interpolation }\end{array}$ & Spline interpolation \\
\hline Shape error $[\mathrm{mm}]$ & 2.139 & 1.379 & 0.471 \\
\hline
\end{tabular}

\section{Conclusion}

This paper raised a method based on fiber sensor array in order to measure the deformation of membrane structure. The first step is to get the strain distribution by FBG sensors. Then the curvature distribution can be calculated. At last, the curved surface is reconstructed by using the surface reconstruction algorithm, and thus we know the deformation of the structure. The results of simulation proved this method's practicability and its high measuring accuracy.

\section{References}

[1] Haiwei Xu, Research on the strain of morphing wings' structure based on distributed[D]. Nanjing University of Aeronautics and Astronautics, 2012. (in Chinese)

[2] Jun Yang, Research on distributed fiber-optic white light Interferometric deformation measuring technique[D]. Harbin Engineering University, 2005. (in Chinese) 
[3] Stephan Rapp, lae-Hyong Kang, Uwe C. Mueller, Jae-Hung Han, Horst Baier, Dynamic shape estimation by modal approach using fiber Bragg grating strain sensors[C]. Sensors and Smart Structures Technologies for Civil, Mechanical, and Aerospace Systms 2007.

[4] Xiaojin Zhu, Lina Jiang, Bing Sun, Hesheng Zhang, Jincong Yi, Shape recongstruction of FBG intelligent flexible structure based on B-spline fitting[J]. Optics and Precision Engeering, 2011,7(19). (in Chinese)

[5] Martin Schroeck, Strain monitoring in steel rock bolts using FBg sensor arrays[J]. In Applications of optical Fiber Sensors, 2000, 4074: 298-304.

[6] Albert Bosse, George C. Kirby, David Bellemore, Alan D. kersey, Tsung Tsai, E. Joesph Friebele, Strain-displacement mapping for a precision truss using Bragg-gratings and identified modeshapes[J], SPIE, 2717(341).

[7] Fengsong Zheng, B spline curve and surface reconstruction based on Genetic Algorithm[D]. Fujian Normal university, 2008. (in Chinese)

[8] Elena Franchini, Implicit shape Reconstruction of Unorganized Points using PDE -based Deformable 3D Manifolds[J]. Numerical Mathematics-Theory Methods and Applications, 2010, 3(4): 405-430.

[9] Gaofeng Meng, Guozhao Wang, Research on Curve and Surface Reconstruction from unorganized Points[D]. Tianjing University, 2005. (in Chinese)

[10]Lincong Fang, Radial basis functions based surface reconstructionb algorithm[J]. Journal of Zhejiang University (Engineering Science), 44(4): 728-731. (in Chinese)

[11] Ruud M. Bolle, Baba C. Vemuri, On Three-Dimensional Surface Reconstruction Methods[J]. IEEE Transactions on Pattern Analysis and Machine Intelligence, 1991, 13(1).

[12]Jincong Yi, Shape Perception and Active Monitoring for Smart Structure Using FBG Sensor Array[D]. Shanghai University, 2014. (in Chinese) 